\title{
Ischaemic Stroke in a Patient With Refractory Idiopathic Thrombocytopenic Purpura: An Unusual Clinical Dilemma
}

\author{
Imranullah Hashmi ${ }^{\mathrm{a}, \mathrm{c}}$, Abdel Magid Bakheit ${ }^{\mathrm{b}}$
}

\begin{abstract}
Idiopathic Thrombocytopenic Purpura (ITP) is commonly associated with bleeding complications, and thrombotic events are very rare. We report a patient with long standing ITP refractory to treatment who developed a large cerebral infarct. The patient's management was complicated by other co-morbidities, a bleeding tendency and recurrent cerebral ischaemia. A careful approach to treatment, based on the understanding of the patho-physiological mechanism of the patient's stroke, his co-morbidity and the estimated risk of haemorrhagic complications was required in this case.
\end{abstract}

Keywords: Idiopathic thrombocytopenic purpura; Ischaemic stroke

\section{Introduction}

Idiopathic thrombocytopenic purpura (ITP) is usually associated with bleeding complications, and thrombotic events including ischaemic stroke are very rare [1-6]. The haemorrhagic manifestations of ITP include purpura, menorrhagia, epistaxis, gingival bleeding, bruising tendency, gastrointestinal haemorrhage and intracranial haemorrhage. Occasionally, ischaemic stroke is the first clinical manifestation in a previously undiagnosed case of ITP $[1,4,6]$. We report here a case of long standing ITP refractory to treatment and presenting with large ischaemic stroke. The patient also suffered

Manuscript accepted for publication February 23, 2012

\footnotetext{
${ }^{\mathrm{a}}$ Specialty Registrar, Neurological Rehabilitation unit, Moseley Hall Hospital, Birmingham, United Kingdom

${ }^{\mathrm{b} C}$ Consultant in Neurological Rehabilitation, Neurological Rehabilitation unit, Moseley Hall Hospital, Birmingham, United Kingdom

${ }^{\mathrm{c}}$ Corresponding author: Imranullah Hashmi, Flat 1, 111 Sandford Road, Birmingham, United Kingdom. Email: hashminet@yahoo.com
}

doi: $10.4021 / \mathrm{jmc} 581 \mathrm{w}$ from gout and other co-morbidities. These, in addition to the risk of bleeding and recurrent cerebral ischaemia, posed significant management challenges.

\section{Case Report}

A 57 year old man was admitted to hospital with severe weakness of the left side of his body, and slurring of his speech. The symptoms occurred suddenly and were preceded by a fall. Clinical examination confirmed the presence of a complete flaccid hemiplegia with facial involvement, left hemispatial neglect and mild dysarthria. There were gross deformities of both knees consistent with chronic osteoarthritis. The rest of the physical examination was entirely normal. A CT head scan was consistent with a diagnosis of a large ischaemic infarct in the territory of the right middle cerebral artery. At the time of admission to the hospital his haemoglobin was $12.9 \mathrm{~g} / \mathrm{dl}$, white cell count was $9 \times 10^{9} / \mathrm{L}$ and platelet count was $15 \times 10^{9} / \mathrm{L}$. His liver and renal function tests were within the normal limits. He was in sinus rhythm and there were no acute changes in the ECG. His carotid Doppler scans were normal.

The patient was known to have ITP for at least 25 years which was refractory to corticosteroids and intravenous immunoglobulin therapy. His usual platelet count had been between $10 \times 10^{9} / \mathrm{L}$ and $20 \times 10^{9} / \mathrm{L}$. Other past medical history included a myocardial infarction three years ago, hypertension and gout. He was not a smoker but had a history of excessive alcohol consumption. His regular medication was allopurinol, low dose aspirin, omeprazole, eplerinone, bisoprolol, ramipril and tramadol. Simvaststin was added on admission for the secondary prevention of stroke. Thrombolysis was not considered appropriate.

Approximately six weeks after hospital admission he developed patecheal rash all over his body and small haemorrhages on the mucosal surfaces. His platelet count at that time was less than $2 \times 10^{9} / \mathrm{L}$. Aspirin was stopped and the blood count was monitored frequently. Four weeks later his platelet count had already improved to $16 \times 10^{9} / \mathrm{L}$. The haemorrhages resolved and he did not have further bleeding.

A few days later he developed flare-up of gout and was 
started on non-steroidal anti-inflammatory medication. A decision was made not to restart aspirin while he was on the non-steroidal anti-inflammatory medication. However, he developed slurring of speech lasting for few hours consistent with a transient ischaemic attack (TIA). The dilemma was whether to re-start the antithrombotic treatment with aspirin. However, on weighing the risks and benefits of using aspirin for the prevention of a further stroke and of the need to continue the non-steroidal anti-inflammatory medication for pain relief and to facilitate mobilisation and physiotherapy we considered it appropriate to withhold aspirin in this case.

\section{Discussion}

ITP, an isolated thrombocytopenia of unknown cause, often results in spontaneous haemorrhage in various tissues and organs. Although intracranial haemorrhage due to ITP is rare, it is one of the most serious complications of ITP. Thrombosis is even more rare in patients with ITP and only a few cases have been previously reported [1, 4, 6-9].

The pathogenesis of intra arterial thrombosis in cases of ITP is complex. Anti-platelet antibodies, which are present in most cases of ITP, cause complement mediated fragmentation of platelets and the release of platelet microparticles (PMP). Although PMP protect against bleeding in cases of ITP, they may also promote intra vascular thrombus formation by causing platelets activation $[10,11]$. The risk of thrombotic complications is also increased by the endothelial damage induced by auto-antibodies directed against antigens present on both platelets and endothelial cells [12].

Thrombotic complications may also occur after splenectomy [13] or result from some therapeutic interventions used for ITP such as treatment with intravenous immunoglobulins which increase blood viscosity, activate platelets or cause vasospasm [14]. Cases of arterial thrombosis in patients with ITP have also been reported after use of Danazol [15]. Furthermore, some patients with ITP are also susceptible to thrombotic complications because they have pro-thrombotic conditions. For example, in their prospective study Diz-kucukkaya et al [16] have found the Anti Phospholipid Syndrome in $17 \%$ of newly diagnosed patients with ITP. Occasionally, thrombotic thrombocytopenic purpura develops after ITP $[17,18]$.

The role of anti-platelet therapy and anticoagulation for the prevention of thrombotic complications in patients with ITP and other hypercoagulable states is not clear and the current reports provide conflicting information [1, 8, 19-21]. It has been argued that anti-platelet agents do not reduce the activity of platelet microparticles, and hence are not effective in management of such cases [6]. Consequently, the management of ischaemic stroke in patients with ITP should be individualized according to the presumed pathophysiologic mechanism of the cerebral infarct, co-morbidity, and estimated risk of haemorrhagic complications [1].

Acute ischaemic stroke requiring urgent thrombolysis is other important management dilemma. As per National Institute of Neurologic Disorders and Stroke inclusion/exclusion criteria, IV thrombolysis with tissue plasminogen activator should not be used if the platelet count is below $100 \times 10^{9} / \mathrm{L}$. In cases of acute myocardial infarction and low platelet count due to ITP, percutaneous coronary intervention has been used [22, 23].

\section{Conclusions}

Although haemorrhage is a common complication of ITP, ischaemic stroke has been reported only rarely. The use of thrombolysis and antithrombotic drugs when ischaemic stroke occurs in a patient with ITP is controversial. In the case reported here the patient had ITP refractory to treatment and developed recurrent cerebral ischemia when aspirin was discontinued because of petechial bleeding into the skin and oral mucosa. The situation was further complicated by his need to receive regular non-steroidal anti inflammatory drugs for the symptomatic treatment of acute gout. These drugs interfere with platelet function but unlike aspirin the duration of their effect is shorter [24]. Their use therefore served a dual function - analgesia and risk reduction of intra vascular thrombosis. At the same time they posed a smaller risk (compared to aspirin) for bleeding. We conclude that the decision as to the optimal treatment regimen in cases such as the one reported here should be considered carefully and the patient's condition should be monitored closely to avoid complications.

\section{References}

1. Theeler BJ, Ney JP. A patient with idiopathic thrombocytopenic purpura presenting with an acute ischemic stroke. J Stroke Cerebrovasc Dis. 2008;17(4):244-245.

2. Neylon AJ, Saunders PW, Howard MR, Proctor SJ, Taylor PR, Northern Region Haematology G. Clinically significant newly presenting autoimmune thrombocytopenic purpura in adults: a prospective study of a population-based cohort of 245 patients. Br J Haematol. 2003;122(6):966-974.

3. Cohen YC, Djulbegovic B, Shamai-Lubovitz O, Mozes B. The bleeding risk and natural history of idiopathic thrombocytopenic purpura in patients with persistent low platelet counts. Arch Intern Med. 2000;160(11):16301638.

4. Phan TG, Owen R. Paradoxical ischaemic stroke in the setting of idiopathic thrombocytopenic purpura. Intern Med J. 2011;41(8):643-644.

5. Cines DB, Bussel JB, Liebman HA, Luning Prak ET. The ITP syndrome: pathogenic and clinical diversity. 
Blood. 2009;113(26):6511-6521.

6. Hak Young Rhee, Hye-Yeon Choi, Sang-Beom Kim, Won-Chul Shin. Recurrent ischaemic stroke in a patient with idiopathic thrombocytopenic purpura. Journal of Thrombosis and Thrombolysis 2010. 30:229-232.

7. Kim JH, Park KU, Chun WJ, Kim SH, Nah DY. Primary percutaneous coronary intervention for acute myocardial infarction with idiopathic thrombocytopenic purpura: a case report. J Korean Med Sci. 2006;21(2):355-357.

8. Tiede MP, Ahn ER, Jy W, Scagnelli T, Bidot CJ, Horstman LL, Jimenez JJ, et al. Life-threatening hypercoagulable state following splenectomy in ITP: successful management with aggressive antithrombotic therapy and danazol. Clin Appl Thromb Hemost. 2005;11(3):347352.

9. Ahn YS, Horstman LL, Jy W, Jimenez JJ, Bowen B. Vascular dementia in patients with immune thrombocytopenic purpura. Thromb Res. 2002;107(6):337-344.

10. Horstman LL, Jy W, Schultz DR, Mao WW, Ahn YS. Complement-mediated fragmentation and lysis of opsonized platelets: ender differences in sensitivity. J Lab Clin Med. 1994;123(4):515-525.

11. Jy W, Horstman LL, Arce M, Ahn YS. Clinical significance of platelet microparticles in autoimmune thrombocytopenias. J Lab Clin Med. 1992;119(4):334-345.

12. Fruchter O, Blich M, Jacob G. Fatal acute myocardial infarction during severe thrombocytopenia in a patient with idiopathic thrombocytopenic purpura. Am J Med Sci. 2002;323(5):279-280.

13. Mohren M, Markmann I, Dworschak U, Franke A, Maas C, Mewes S, Weiss G, et al. Thromboembolic complications after splenectomy for hematologic diseases. Am J Hematol. 2004;76(2):143-147.

14. Emerson GG, Herndon CN, Sreih AG. Thrombotic complications after intravenous immunoglobulin therapy in two patients. Pharmacotherapy. 2002;22(12):16381641.
15. Alvarado RG, Liu JY, Zwolak RM. Danazol and limbthreatening arterial thrombosis: two case reports. J Vasc Surg. 2001;34(6):1123-1126.

16. Diz-Kucukkaya R, Hacihanefioglu A, Yenerel M, Turgut M, Keskin H, Nalcaci M, Inanc M. Antiphospholipid antibodies and antiphospholipid syndrome in patients presenting with immune thrombocytopenic purpura: a prospective cohort study. Blood. 2001;98(6):1760-1764.

17. Stein RS, Flexner JM. Idiopathic thrombocytopenic purpura during remission of thrombotic thrombocytopenic purpura. South Med J. 1984;77(12):1599-1601.

18. Olenich M, Schattner E. Postpartum thrombotic thrombocytopenic purpura (TTP) complicating pregnancy-associated immune thrombocytopenic purpura (ITP). Ann Intern Med. 1994;120(10):845-847.

19. Rosove MH, Brewer PM. Antiphospholipid thrombosis: clinical course after the first thrombotic event in 70 patients. Ann Intern Med. 1992;117(4):303-308.

20. Peters TG, Lewis JD, Flip DJ, Morris L. Antithrombin III deficiency causing postsplenectomy mesenteric venous thrombosis coincident with thrombocytopenia. Ann Surg. 1977;185(2):229-231.

21. Leuzzi RA, Davis GH, Cowchock FS, Murphy S, Vernick JJ. Management of immune thrombocytopenic purpura associated with the antiphospholipid antibody syndrome. Clin Exp Rheumatol. 1997;15(2):197-200.

22. Kikuchi S, Hayashi Y, Fujioka S, Kukita H, Ochi N. [A case of intracoronary stent implanted for acute myocardial infarction in an elderly patient with idiopathic thrombocytopenic purpura]. Nihon Ronen Igakkai Zasshi. 2002;39(1):88-93.

23. Fong MC, Chen KC, Leu HB, Chen LC. Coronary revascularization in a patient with immune thrombocytopenic purpura. J Chin Med Assoc. 2006;69(9):436-438.

24. McQueen EG, Facoory B, Faed JM. Non-steroidal antiinflammatory drugs and platelet function. N Z Med J. 1986;99(802):358-360. 